\title{
Erratum to the article Embeddings from the point of view of immersion theory: Part I
}

\author{
MICHAEL WEISS
}

This repairs an error in the proof of Theorem 5.2 in [1].

57R40; 57R42

It was pointed out to me recently by Victor Tourtchine (aka Turchin) that the second half of the proof of Theorem 5.2 in [1] is faulty. The theorem does not occupy a central position in [1], but it does make an illuminating connection between functor calculus and sheaf theory. It is restated below, after some definitions. A revised proof follows the statement. I am indebted to Victor Tourtchine for help.

Let $M$ be a smooth manifold without boundary, $\mathcal{O}$ the poset of open subsets of $M$, and $\mathcal{J}_{k}$ for $k \geq 1$ the Grothendieck topology on $\mathcal{O}$ in which a collection $\left\{V_{i} \rightarrow W\right\}$ of inclusions in $\mathcal{O}$ is a covering of $W$ if $\bigcup_{i} V_{i}^{k}=W^{k}$. See [1] for homotopy sheaf with respect to $\mathcal{J}_{k}$ (Section 0), good cofunctor from $\mathcal{O}$ to Spaces (Section 1), polynomial cofunctor of degree $\leq k$ (Section 2) and the subposet $\mathcal{O} k$ of $\mathcal{O}$ (Section 3).

Theorem 5.2 A good cofunctor $F$ from $\mathcal{O}$ to Spaces is polynomial of degree $\leq k$ if and only if it is a homotopy sheaf with respect to the Grothendieck topology $\mathcal{J}_{k}$.

The first half of the proof (homotopy sheaf $\Rightarrow$ polynomial) given in [1] appears to be correct. In the second half of the proof, for $k>1$, the choice of open covering $\varepsilon$ is ill-considered, with the consequence that Lemma 4.2 is not applicable despite being invoked.

For a better second half, fix $W$ in $\mathcal{O}$ and a covering $\left\{V_{i} \mid i \in S\right\}$ of $W$ in the $\mathcal{J}_{k}$ sense. Assume that $F$ is polynomial of degree $\leq k$. The goal is to show that the canonical map

$$
F(W) \longrightarrow \operatorname{holim}_{R} F\left(\bigcap_{i \in R} V_{i}\right)
$$


is a homotopy equivalence, where $R$ runs through the nonempty finite subsets of $S$. Choose an exhaustion of $W$ by open subsets

$$
W_{1} \subset W_{2} \subset W_{3} \subset \cdots
$$

such that $W_{j}$ has compact closure in $W_{j+1}$, for all $j \geq 1$. Since $F$ is a good cofunctor, we may replace (1) by

$$
\underset{j}{\operatorname{holim}} F\left(W_{j}\right) \longrightarrow \underset{R}{\operatorname{holim}} \operatorname{holim} F\left(\bigcap_{j \in R}\left(V_{i} \cap W_{j}\right)\right) .
$$

In the target, we may interchange the order in which the homotopy inverse limits are formed (Fubini for homotopy limits). Then the homotopy invariance properties of homotopy inverse limits (here over the variable $j$ ) come to our aid, and it only remains to show that

$$
F\left(W_{j}\right) \longrightarrow \operatorname{holim}_{R} F\left(\bigcap_{i \in R}\left(V_{i} \cap W_{j}\right)\right)
$$

is a homotopy equivalence for fixed $j$. As $j$ is fixed, we write $W^{\prime}:=W_{j}$ and $V_{i}^{\prime}:=V_{i} \cap W_{j}$, so that $\left\{V_{i}^{\prime} \mid i \in S\right\}$ is a covering of $W^{\prime}$ in the $\mathcal{J}_{k}$ sense.

Because $W^{\prime}$ has compact closure in $W$, there exists a finite subset $S^{\prime}$ of $S$ such that $\left\{V_{i}^{\prime} \mid i \in S^{\prime}\right\}$ is still a covering of $W^{\prime}$ in the $\mathcal{J}_{k}$ sense. Even better: for each $i \in S^{\prime}$ we can choose $V_{i}^{\prime \prime}$ open in $W^{\prime}$ such that the closure of $V_{i}^{\prime \prime}$ is contained in $V_{i}^{\prime}$ and $\left\{V_{i}^{\prime \prime} \mid i \in S^{\prime}\right\}$ is still a covering of $W^{\prime}$ in the $\mathcal{J}_{k}$ sense.

Now let $\varepsilon$ (redefined) be an open covering of $W^{\prime}$, in the familiar $\mathcal{J}_{1}$ sense, such that every open set of $\varepsilon$ which has nonempty intersection with some $V_{i}^{\prime \prime}$ for $i \in S^{\prime}$ is contained in $V_{i}^{\prime}$ for the same $i$. Let $U$ be an object of $\mathcal{O} k$ which is $\varepsilon$-small (every connected component of $U$ is contained in some open set of $\varepsilon$ ) and such that $U \subset W^{\prime}$. Then $U$ is contained in $V_{i}^{\prime}$ for some $i \in S^{\prime}$. (Choose a finite $X \subset U$ such that every component of $U$ contains exactly one element of $X$. Then $X \subset V_{i}^{\prime \prime}$ for some $i \in S^{\prime}$ and so $U \subset V_{i}^{\prime}$ for the same $i$.)

Let $E$ be the restriction of $F$ to $\mathcal{O} k$. Define $\varepsilon E^{!}$as in Section 3 of [1] just before Theorem 3.9, where $\varepsilon$ is the covering of $W^{\prime}$ just constructed. Up to equivalence, $F$ and $\varepsilon E^{!}$are the same. By Lemma 4.2 of [1], whose applicability has just been verified, the canonical map

$$
\varepsilon E^{!}(W) \longrightarrow \operatorname{holim}_{R} \varepsilon E^{!}\left(\bigcap_{i \in R} V_{i}\right)
$$

is a homotopy equivalence. Here again, $R$ runs through the finite nonempty subsets $R$ of $S$. 


\section{References}

[1] M Weiss, Embeddings from the point of view of immersion theory: Part I, Geom. Topol. 3 (1999) 67-101 MR1694812

Department of Mathematics, University of Aberdeen

Aberdeen AB24 3UE, UK

m. weiss@abdn.ac.uk

http://www. maths.abdn.ac.uk/ mweiss/

Received: 22 October 2010 